\title{
Influência do Fotoperíodo no Parasitismo de Trichogramma galloi Zucchi (Hymenoptera: Trichogrammatidae) em Ovos de Diatraea saccharalis (Fabricius) (Lepidoptera: Crambidae)
}

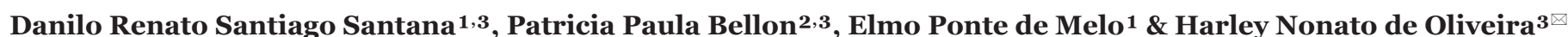

1. UNIGRAN - Centro Universitário da Grande Dourados, e-mail: d santana87@yahoo.com.br, epmeloagro@yahoo.com.br. 2.Universidade Federal da Grande Dourados, e-mail: phatriciabellon@yahoo.com.br. 3. Embrapa - CPAO, e-mail: harley.oliveira@embrapa.br (Autor para correspondência ${ }^{\bowtie}$ )

EntomoBrasilis 6(2): 165-167 (2013)

Resumo. As espécies de Trichogramma constituem um importante grupo de parasitoides que atacam, principalmente, ovos de lepidópteros. A qualidade e o desempenho desse inimigo natural podem ser influenciados por alguns fatores, como hospedeiro alternativo utilizado nas criações massais, temperatura e diferenças de fotoperíodo. Nesse sentido, o objetivo desse trabalho foi avaliar o número de ovos parasitados, \% de fêmeas que parasitaram, \% emergência, razão sexual e número de indivíduos de Trichogramma galloi Zucchi em os ovos de Diatraea saccharalis (Fabricius), na presença e ausência de luminosidade. O delineamento experimental utilizado foi inteiramente casualizado com dois tratamentos (claro e escuro) com 60 repetições, cada uma dessas foi constituída por 40 ovos de $D$. saccharalis que foram colocados em tubo de vidro com uma fêmea de $T$. galloi com 24 horas de idade, previamente alimentadas com mel. O experimento foi conduzido à $25^{\circ} \mathrm{C} \pm 2^{\circ} \mathrm{C} \mathrm{e} 70 \pm 10 \%$ umidade relativa. Em cada um dos tratamentos, o parasitoide foi removido após 12 horas. O maior número de ovos parasitados $(12,9)$ e \% de fêmeas que parasitaram (83,3\%) foi observado no período com luz. Em relação à emergência, razão sexual e número de indivíduos por ovo, não houve diferença estatística. Os resultados demonstraram que a presença de luminosidade é essencial para esse agente de controle biológico.

Palavras-chave: Cana-de-açúcar; Controle biológico; Parasitoide.

Influence of Photoperiod on Parasitism of Trichogramma galloi Zucchi (Hymenoptera: Trichogrammatidae) on Eggs of Diatraea saccharalis (Fabricius) (Lepidoptera: Crambidae)

Abstract. Trichogramma species are an important group of parasitoids that attack especially lepidopteran eggs. The quality and performance of this natural enemy may be influenced by factors such as alternative host used in the massal rearing, and differences in the temperature and photoperiod. Based on this, this study aimed to evaluate the number of parasitized eggs, \% of females that parasitized, \% emergence, sex ratio and number of individuals of Trichogramma galloi Zucchi per egg of Diatraea saccharalis (Fabricius) in the presence and absence of light. The experimental design was completely randomized with two treatments (light and dark) and 6o replications, being each experimental unit composed by 40 eggs of $D$. saccharalis which were placed in a glass tube with one female of $T$. galloi with age of 24 hours, previously fed with honey. The experiment was carried out at $25 \pm 2{ }^{\circ} \mathrm{C}$ and $70 \pm 10 \%$ relative humidity. In each one of the treatments, the parasitoid was removed from the tube after 12 hours. The highest number of parasitized eggs (12.9) and \% of females that parasitized (83.3\%) was observed during the period with light. For the emergence, sex ratio and number of individuals per egg, there was no statistical difference. The results showed that the presence of light is essential for this biological control agent.

Keywords: Biological control; Parasitoid; Sugarcane.

Brasil se destaca por ser o maior produtor de canade-açúcar (Saccharum officinarum L.) e por dominar todos os estágios da tecnologia de produção de açúcar e etanol (Willadino et al. 2011) com produção de 662 milhões de toneladas, em uma área aproximada de 9,1 milhões de hectares plantados (IBGE 2012).

Apesar da adaptação da cultura ao clima do país, ela enfrenta problemas fitossanitários, principalmente com a incidência de insetos-praga tais como a broca da cana-de-açúcar Diatraea saccharalis (Fabricius) (Lepidoptera: Crambidae), a qual diminui a produtividade, causando prejuízo aos produtores (BoIÇA JÚNIOR et al. 1997).

Atualmente o método de controle mais eficiente para essa praga se baseia no uso do controle biológico através do parasitoide larval Cotesia flavipes (Cameron) (Hymenoptera: Braconidae) (PEREIRABARros et al. 2005) e do parasitoide de ovos Trichogramma galloi Zucchi (Hymenoptera: Trichogrammatidae) (LimeIRA \& RAFIKOV 2010).

Espécies do gênero Trichogramma apresentam ampla distribuição geográfica e um grande número de hospedeiros (Zucchi \& Monteiro 1997); além disso, são de grande interesse, principalmente pelo fato de atuarem na fase de ovo, ou seja, antes mesmo da praga causar danos à cultura (LimEIRA \& RAFIKOV 2010) e devido à facilidade de criação em laboratório (OLIVEIRA et al. 2007).

No entanto, a qualidade e aspectos biológicos de Trichogramma podem ser influenciados por alguns fatores, como o hospedeiro alternativo utilizado nas criações massais (OliveIRA et al. 2005), a temperatura (Pratissoli et al. 2004) e o fotoperíodo (Shirazi 2006).

Agência(s) de Fomento: CNPq, FUNDECT e EMBRAPA 
T Apesar da importância de T. galloi na cultura da cana-de-açúcar e do mesmo ser utilizado em programas de controle biológico, as informações sobre o efeito da ausência/presença de luminosidade no parasitismo desse inimigo natural são escassas. Nesse sentido, o objetivo do presente trabalho foi avaliar o efeito da presença e ausência de luz sobre o comportamento desse parasitoide em ovos de D. saccharalis.

O experimento foi realizado no Laboratório do Controle Biológico da Embrapa Agropecuária Oeste, em Dourados, Mato Grosso do Sul.

Para a realização dos ensaios, cada tratamento (claro e escuro) foi constituído por sessenta repetições. Cada repetição foi constituída por uma massa de quarenta ovos de $D$. saccharalis com menos de 24 horas de idade que foi oferecida a uma fêmea de T. galloi com 24 horas de idade, previamente alimentada com mel. Essas fêmeas, de cada repetição, foram individualizadas em tubos de vidro $(2,5$ $\mathrm{cm}$ de diâmetro x 8,5 cm de comprimento), vedados com papel filme de PVClaminado, perfurado com alfinete entomológico para aeração. Posteriormente, os tubos permaneceram em câmaras climatizadas (BOD), com temperatura de $25 \pm 2{ }^{\circ} \mathrm{C}, \mathrm{UR} 7 \mathrm{O} \pm 10 \%$, mantidos por 12 horas na presença ou ausência de luminosidade. Após exposição dos ovos ao parasitismo por 12 horas, as fêmeas de T. galloi foram retiradas dos tubos e as massas de ovos foram mantidas nas mesmas condições até o final das avaliações, onde foi verificado o número de ovos parasitados, porcentagem de fêmeas que parasitaram, porcentagem de emergência, razão sexual e número de indivíduos por ovo.

O delineamento experimental utilizado foi inteiramente casualizado, sendo que as médias foram submetidas à análise de variância e comparadas entre si pelo teste $t$, utilizando-se o programa estatístico Assistat 7.6 Beta.

Observou-se maior número de ovos parasitados por T. galloi no período onde houve luminosidade $(12,9)$ quando comparado ao período escuro $(3,0)$ (Tabela 1). Shirazi (2006) também verificou para Trichogramma chilonis Ishii (Hymenoptera: Trichogrammatidae) em ovos de Corcyra cephalonica (Stainton)
(Lepidoptera: Pyralidae) a influência da luminosidade no número de ovos parasitados. BoтelHo (1997) relata que fêmeas de T. galloi parasitaram maior número de ovos de $D$. saccharalis durante o dia, demonstrando seu hábito diurno, à semelhança a outras espécies desse gênero. Os resultados encontrados no presente trabalho confirmam que as fêmeas de T. galloi preferem parasitar ovos na presença de luz.

Em relação ao número de fêmeas que parasitaram, observaramse diferenças significativas no período diurno (83,3\%) quando comparado ao período noturno (25\%) (Tabela 1), demonstrando que a presença de luz é fator preponderante para que ocorra parasitismo de T. galloi.

Para a emergência, não se observou diferenças significativas entre os fotoperíodos, obtendo-se valores de $67,5 \%$ no período diurno e de 55,4\% no período noturno. Lima FiLHo \& Lima (2003) também não verificaram diferenças significativas na emergência de T.galloi em ovos D. saccharalis e Anagasta kuehniella (Zeller) (Lepidoptera Pyralidae), demonstrando que uma vez parasitados, o tipo de hospedeiro utilizado não afeta a emergência.

O número médio de adultos de T. galloi, emergidos por ovo de D. saccharalis, também não foi afetado pela presença/ausência de luminosidade (2,0 e 1,8 respectivamente) (Tabela 1). PEREIRABARros et al. (2005) avaliando os aspectos biológicos de T. galloi, criados em ovos de $D$. saccharalis, também constataram valores semelhantes de indivíduos emergidos por ovo.

Quando se avaliou a razão sexual, não foram observadas diferenças significativas entre os tratamentos (Tabela 1), sendo os valores semelhantes aos relatados por SALES JR. (1992) nessa mesma espécie e por ZART et al. (2012) para Trichogramma pretiosum Riley (Hymenoptera: Trichogrammatidae) em ovos de A. kuehniella.

Com base nas características avaliadas, conclui-se que a presença de luz é essencial para o parasitismo de $T$. galloi em ovos de $D$. saccharalis.

Tabela 1 - Número de ovos parasitados, (\%) fêmeas que parasitaram, emergência (\%), razão sexual, número de indivíduos por ovo de Trichogramma galloi em ovos da Diatraea saccharalis a $25 \pm 2^{\circ} \mathrm{C}$, UR $70 \pm 10 \%$, mantidos por 12 horas de fotofase e 12 horas de escotofase.

\begin{tabular}{lccccc}
\hline & $\begin{array}{c}\mathbf{N}^{\circ} \text { de ovos } \\
\text { parasitados }\end{array}$ & $\begin{array}{c}\text { \% fêmeas que } \\
\text { parasitaram }\end{array}$ & \% emergência & Razão Sexual & $\begin{array}{c}\mathbf{N}^{\circ} \text { de indivíduos } \\
\text { por ovo }\end{array}$ \\
\hline Claro & $12,9 \mathrm{a}$ & $83,3 \mathrm{a}$ & $67,5 \mathrm{a}$ & $0,85 \mathrm{a}$ & $2,0 \mathrm{a}$ \\
Escuro & $3, \mathrm{ob}$ & $25, \mathrm{ob}$ & $55,4 \mathrm{a}$ & $0,87 \mathrm{a}$ & $1,8 \mathrm{a}$ \\
\hline
\end{tabular}

Médias seguidas de mesma letra na coluna, não diferem entre si pelo teste de $\mathrm{t}$.

\section{REFERÊNCIAS}

Boiça Júnior, A.L., Lara, F.M. \& M.P. Bellodi, 1997. Influência de variedades de cana-de-açúcar, incorporadas em dieta artificial, no desenvolvimento de Diatraea saccharalis (Fabr.) e no seu parasitismo por Cotesia flavipes (Cam.). Anais da Sociedade Entomológica do Brasil, 26: 537-542.

Botelho, P.S.M., 1997. Eficiência de Trichogramma em campo, p. 303-318. In: Parra, J.R.P. \& R.A. Zucchi (Eds.). Trichogramma e o controle biológico aplicado. Piracicaba, FEALQ, 324p.

IBGE, 2012. Levantamento Sistemático da Produção Agrícola. Rio de Janeiro, 25: 127. Disponível em: <http://www.ibge. gov.br/home/estatistica/indicadores/agropecuaria/lspa/ default publ completa.shtm>. Acesso em: 23 jul. 2012.

Lima Filho, M. \& J.O.G. Lima, 2003. Efeito de escamas de Diatraea saccharalis (Fabr) na produção de Trichogramma galloi Zucchi criado em ovos de Anagasta kuehniella (Zeller). Revista Universidade Rural, Série Ciências da Vida, 22: 2331.
Limeira, E.H. \& M. Rafikov, 2010. Dinâmica de interação da praga da cana-de-açúcar com seu parasitoide Trichogramma galloi. In: Conferência Brasileira de Dinâmica, Controle e Aplicações, 10., 2011, Serra Negra. [Anais...]. [S.l.], ABCN, SBA, SBMAC, p. 238-242. DINCON'10.

Oliveira, H.N., C.A. Colombi, D. Pratissoli, E.P. Pedruzzi \& L.P. Dalvi, 2005. Capacidade de parasitismo de Trichogramma exiguum Pinto \& Platner, 1978 (Hymenoptera: Trichogrammatidae) criado em dois hospedeiros por diversas gerações. Ciência Agrotecnologia, 29: 284-288.

Oliveira, H.N., D. Pratissoli, C.A. Colombi, R.A. Polanczyk \& L.P. Dalvi, 2007. Tabela de vida de fertilidade de Trichogramma exiguum (Hymenoptera: Trichogrammatidae). Idesia, 25: 73-76.

Pereira-Barros, J.L., S.M.F. Broglio-Micheletti, A.J.N. Santos, L.W.T. Carvalho, L.H.T. Carvalho \& C.J.T. Oliveira, 2005. Aspectos biológicos de Trichogramma galloi Zucchi, 1988 (Hymenoptera: Trichogrammatidae) criados em ovos de Diatraea saccharalis (Fabricius, 1794) (Lepidoptera: Crambidae). Ciência Agrotecnologia, 29: 714-718. 
Pratissoli, D., H.N. Oliveira, S.M.J. Vieira, R.C. Oliveira \& H.B. Zago, 2004. Efeito da disponibilidade de hospedeiro e de alimento nas características biológicas de Trichogramma galloi Zucchi (Hymenoptera: Trichogrammatidae). Revista Brasileira de Entomologia, 48: 101-104.

Sales JR., 1992. Bioecologia de Trichogramma galloi Zucchi, 1988 no hospedeiro natural Diatraea saccharalis (Fabricius, 1794) e em hospedeiros alternativos. 97p. Tese (Doutorado em Entomologia) - Escola Superior de Agricultura "Luiz de Queiroz", Universidade de São Paulo, Piracicaba.

Shirazi, J., 2006. Effect of Temperature and Photoperiod on the Biological Characters of Trichogramma chilonis Ishii (Hymenoptera: Trichogrammatidae) Pakistan Journal of Biological Sciences, 9: 820-824.

Willadino, L., R.A. Oliveira Filho, E.A. Silva Junior, A.G. Neto \& T.R. Camara, 2011. Estresse salino em duas variedades de cana-de-açúcar: enzimas do sistema antioxidativo e fluorescência da clorofila. Revista Ciência Agronômica, 42: 417-422.
Zart, M., O. Bernardi, A.M. Nunes, F.S. Andersson, S. ManfrediCoimbra, G.R. Busato \& M.S. Garcia, 2012. Influência do Fotoperíodo e da Densidade de Ovos de Anagasta kuehniella (Zeller) Sobre Aspectos Biológicos e Paraistismo de Ovos por Trichogramma pretiosum Riley. EntomoBrasilis, 5: 115-119.

Zucchi, R.A. \& R.C. Monteiro, 1997. O gênero Trichogramma na América do Sul, p. 41-66. In: Parra, J.R.P. \& R.A. Zucchi (Eds.). Trichogramma e o controle biológico aplicado. Piracicaba, FEALQ, 324p.

\section{Recebido em: 01/o8/2012}

Aceito em: 28/01/2013

\section{Como citar este artigo:}

Santana, D.R.S., P.P. Bellon, E.P. Melo \& H.N. Oliveira, 2013. Influência do Fotoperíodo no Parasitismo de Trichogramma galloi Zucchi (Hymenoptera: Trichogrammatidae) em Ovos de Diatraea saccharalis (Fabricius) (Lepidoptera: Crambidae). EntomoBrasilis, 6(2): 165-167. Acessível em: http://www.periodico.ebras.bio.br/ojs/index.php/ebras/article/view/263. doi:10.12741/ebrasilis.v6i2.263

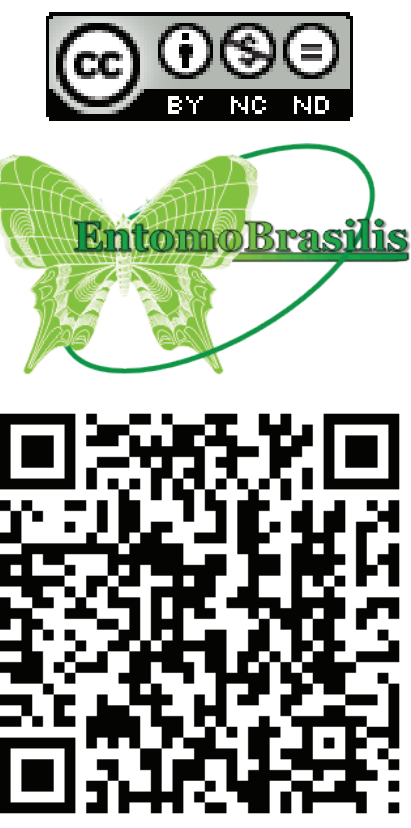

